\title{
Desenvolvimento e resultados de uma atividade de confecção do sistema solar em escala real de distâncias e tamanhos em uma escola de ensino médio da rede pública estadual do Espírito Santo
}

\author{
Silva, T. P. ${ }^{1 *} ;$ Bisch, S.M. ${ }^{2}$; \\ 1 Centro Estadual de Ensino Médio em Tempo Integral Maura Abaurre, Vila Velha, ES, Brasil. \\ 2 Departamento de Física, Universidade Federal do Espírito Santo, Vitória, ES, Brasil.
}

*e-mail: thiagopereiradasilva@yahoo.com.br

\begin{abstract}
Resumo
$\mathrm{Na}$ aplicação de uma sequência didática denominada "Nossa Posição no Universo" com 40 alunos de uma escola da rede pública estadual do Espírito Santo, foi verificada, mediante a análise dos dados do questionário inicial, a total falta de dimensionamento do Sistema Solar. A aplicação dessa sequência didática fez parte de uma disciplina eletiva, denominada O Céu, nossa janela para o Universo, que tem como objetivo principal o ensino de Astronomia e incluiu a construção de um modelo do Sistema Solar em escala real de tamanhos e distâncias utilizando material de baixo custo e realizada no pátio da escola. Inicialmente os alunos confeccionavam seus sistemas solares, em grupos de no máximo 5 estudantes, utilizando seus conhecimentos prévios. Após a execução desse primeiro momento um debate foi realizado. As dimensões reais de tamanho e distâncias foram apresentadas. Observou-se que os alunos tinham certa noção de tamanho, mas não sabiam compará-los. Na segunda etapa da atividade, tomando o Sol como uma esfera de isopor de $20 \mathrm{~cm}$, retornamos ao pátio e construímos o Sistema Solar em escala real de tamanhos e distâncias. Ao comparar o sistema real (montado em escala real) com os sistemas de conhecimentos prévios, os alunos puderam observar as quão equivocadas estavam as suas concepções iniciais. O próximo passo foi um debate da atividade e uma apresentação a comunidade escolar.
\end{abstract}

\begin{abstract}
In the application of a so-called educational sequence "Our Position in the Universe" with 40 pupils of a school of the state public net of the Holy Spirit, the total lack was checked, by means of the analysis of the data of the initial questionnaire, of dimensionamento of the Solar System. The application of this educational sequence made part of an elective, so-called discipline O Céu, nossa janela para o Universo, what takes the teaching of Astronomy as a main objective and included the construction of a model of the Solar System in real scale of sizes and distances using material of low cost and carried out in the patio of the school. Initially the pupils were making his solar systems, in groups of at most 5 students, using his prior knowledges. After the execution of this first moment a discussion was carried out. The real dimensions of size and distances were presented. It was noticed that the pupils had certain notion of size, but they could not compare them. In the second stage of the activity, sunbathing like a sphere of $20 \mathrm{~cm}$ polystyrene, we return to the patio and build the Solar System in real scale of sizes and distances. While comparing the real system (mounted in real scale) with the systems of prior knowledges, the pupils could notice it how mistaken his initial conceptions were. The near step was a discussion of the activity. The next step was a discussion of the activity and a presentation to the school Community.
\end{abstract}

Keywords (Palavras chaves):Sistema Solar, Ensino de Astronomia, Instrumentação de Ensino.

\section{Introdução}

O intuito deste trabalho é apresentar alguns dos resultados obtidos em termos de mudanças nas concepções de um grupo de estudantes do Ensino Médio com relação ao Céu e ao Universo resultantes de sua participação em uma sequência didática que teve como tema central a nossa posição no universo. $O$ grupo era formado por 40 estudantes de uma escola pública estadual. A sequência, intitulada "Nossa Posição no Universo" (SILVA, 2015), teve como principais objetivos:

1. Evidenciar que o céu é nossa janela para o universo, que os principais tipos de objetos que compõem o nosso universo visível, tais como planetas, estrelas e galáxias, podem ser observados e reconhecidos no céu noturno, mesmo a vista desarmada. 
2. Ensinar noções básicas acerca dos principais componentes do universo visível: planetas, seus satélites, estrelas e galáxias, abordando sua natureza, composição e escalas de tamanho e distâncias.

3. Abordar a estrutura espacial do Universo: a profundidade do céu, como se organizam no espaço os principais componentes do universo visível e qual é nossa posição dentro dele.

4. Ensinar acerca da idade do universo, por meio de uma comparação entre o tempo de existência do universo com o tempo de existência da espécie humana na Terra e com o de uma vida humana.

A sequência foi estruturada de acordo com a seguinte ordenação de atividades:

1. Aplicação de um questionário inicial composto por 19 questões, sendo 12 abertas ou semiabertas e 7 fechadas, visando o levantamento das concepções iniciais dos estudantes acerca dos temas e conceitos a serem trabalhados durante a sequência;

2. Apresentação de dois vídeos motivadores: "Potências de 10" (EAMES; EAMES, 1977) e "O Universo Conhecido" (HOFFMAN; EMMART, 2009);

3. Desenvolvimento da atividade prática "Observando o céu noturno a olho nu";

4. Debate em torno da atividade "Observando o céu noturno a olho nu", utilizando o programa Stellarium, com a identificação de um planeta (Saturno) dentre as "estrelas" mais brilhantes marcadas pelos alunos na atividade anterior;

5. Realização da oficina "Confeccionando a Constelação do Cruzeiro do Sul" em que é montado um modelo tridimensional da constelação em escala real de distâncias;

6. Construção do "Sistema Solar em escala real de distâncias";

7. Atividade "Observação do Céu noturno com telescópio";
8. Visita a um planetário para participar de uma sessão abordando o tema da nossa posição no universo;

9. Aplicação de um questionário final, semelhante ao inicial, na tentativa de identificar uma esperada evolução nas concepções dos estudantes;

10. Realização de uma entrevista semiestruturada visando uma avaliação da aprendizagem e de eventuais mudanças de concepção e atitude com relação ao céu e ao universo.

A sequência didática e as atividades foram desenvolvidas entre os meses de junho a novembro de 2017 na eletiva denominada O Céu, nossa janela para o Universo, que teve como objetivo principal o ensino de Astronomia. Os encontros da eletiva, ocorriam nas quintas-feiras e correspondiam a duas aulas de 50 minutos.

O trabalho relata a mudança das concepções prévias dos alunos diante das dimensões de tamanho e de distância do Sistema Solar, que corresponde a atividade 6 da sequência didática "Nossa posição no Universo" (SILVA, 2015). Essa atividade inclui a construção de um modelo do Sistema Solar em escala real de tamanhos e distâncias utilizando material de baixo custo e realizada no pátio da escola. A sequência foi apresentada assim aos estudantes:

1. Confecção do Sistema Solar dos alunos no pátio da escola;

2. Debate;

3. Apresentação do vídeo: "To Scale: The Solar System"

4. Apresentação das escalas;

5. Confecção do Sistema Solar em escala real de tamanhos e distâncias;

6. Debate;

7. Apresentação do Sistema Solar a comunidade escolar.

Após as confecções dos dois Sistemas Solares, digamos, um de conhecimento prévio e outro após a execução das atividades, os alunos puderam observar 
o quão equivocadas estavam as suas concepções iniciais.

\section{A metodologia empregada}

Inicialmente os alunos confeccionaram seus sistemas solares, em grupos de 5 estudantes, utilizando seus conhecimentos prévios. Neste primeiro momento, os alunos receberam um giz e massa de modelar. Foi instituído um ponto fixo de referência. No ponto fixo, estava uma bola de basquete, representando o Sol, assim, os estudantes fizeram suas marcações das distâncias dos planetas, em relação ao Sol, bem como o tamanho deles. Após a execução desse primeiro momento um debate foi realizado.

No encontro seguinte, assistimos o vídeo To Scale: The Solar System, disponível em: https://www.youtube.com/watch?time continue=3\&v=z R3lgc3Rhfg e realizamos um debate. Após esse processo, as reais dimensões foram apresentadas aos alunos, bem como o processo matemático de escalonar as distâncias e os tamanhos, tendo como base uma esfera de isopor de $20 \mathrm{~cm}$. Cada grupo ficou responsável por um planeta recebendo um pedaço de massa epóxi e uma régua escolar para confeccionar os tamanhos dos planetas. A tabela 1, demonstra as dimensões do Sistema Solar em escala real de tamanho e distância calculados em conjunto com os alunos.

Tabela 01: Dimensões do Sistema Solar em escala real de tamanho e distância

\begin{tabular}{c|c|c|c|c}
\hline Objeto & $\begin{array}{l}\text { Real } \\
\text { Diâmetro } \\
(\mathbf{k m})\end{array}$ & $\begin{array}{l}\text { Real } \\
\text { Distância } \\
(\mathbf{m i l h o ̃ e s} \\
\text { de km) }\end{array}$ & $\begin{array}{l}\text { Tamanho } \\
\text { escalonado } \\
(\mathbf{c m})\end{array}$ & $\begin{array}{l}\text { Distância } \\
(\mathbf{m})\end{array}$ \\
\hline Sol & 1392000 & & 20,0 & \\
\hline Mercúrio & 4880 & 57,910 & 0,07 & 8,30 \\
\hline Vênus & 12104 & 108,16 & 0,17 & 15,50 \\
\hline Terra & 12742 & 149,6 & 0,18 & 21,50 \\
\hline Marte & 6780 & 228,0 & 0,10 & 32,80 \\
\hline Júpiter & 139822 & 778,4 & 2,0 & 111,85 \\
\hline Saturno & 116464 & 1427,0 & 1,7 & 205,00 \\
\hline Urano & 50724 & 2869,6 & 0,7 & 412,30 \\
\hline Netuno & 49248 & 4496,6 & 0,7 & 646,10 \\
\hline
\end{tabular}

A figura 1, abaixo, ilustra o momento das confecções.

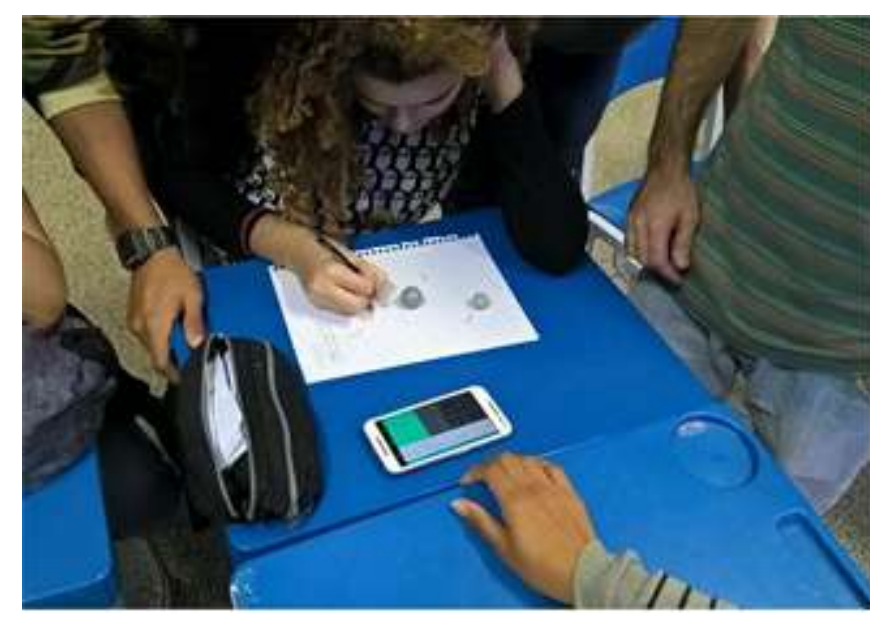

Figura 1: Alunos confeccionando o tamanho dos planetas em escala real

No encontro seguinte, os alunos construíram definitivamente o Sistema Solar. As dimensões do nosso pátio permitiram até o planeta Saturno. As figuras 2 e 3 ilustram essa situação.

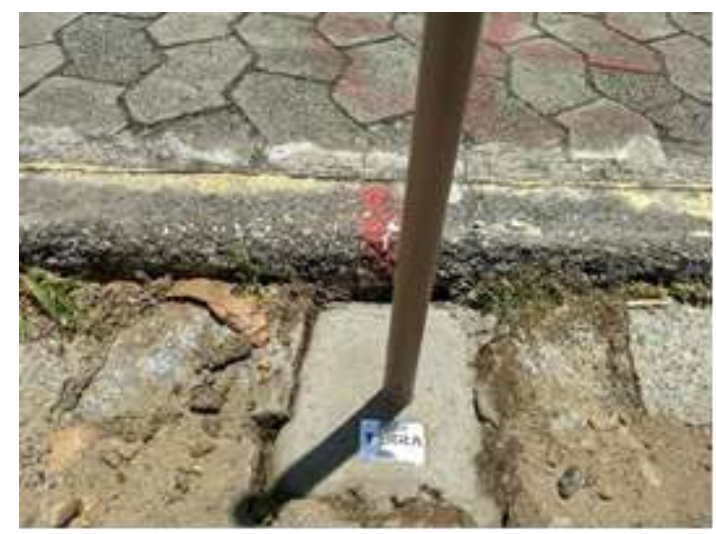

Figura 2: Montagem do Sistema Solar em escala real de distância e tamanho

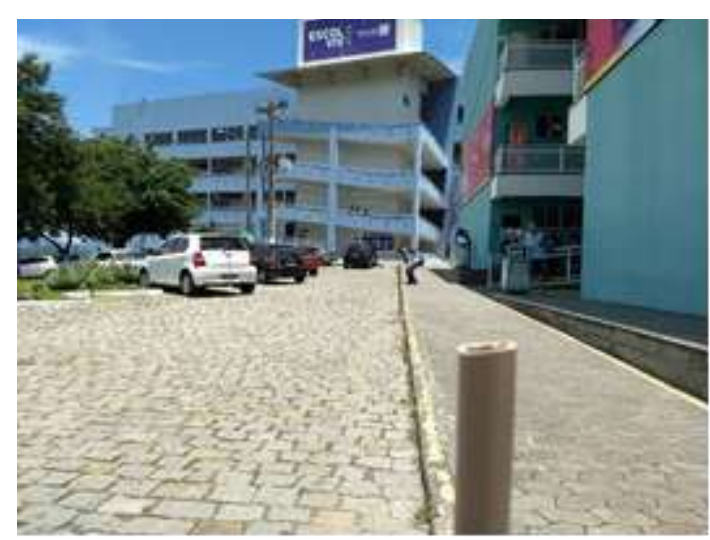

Figura 3: Montagem do Sistema Solar em escala real de distância e tamanho

Após a execução, houve mais um encontro na qual percorremos pelo Sistema Solar e discutimos suas dimensões e particularidades de cada planeta. No 
planeta Terra, os alunos puderam verificar que o disco solar era muito próximo do disco da bola isopor, indicando o acerto no processo das escalas. Os alunos, após todo o debate, foram convidados a apresentar o Sistema Solar à comunidade escolar. As figuras 4 e 5 , ilustram este momento.

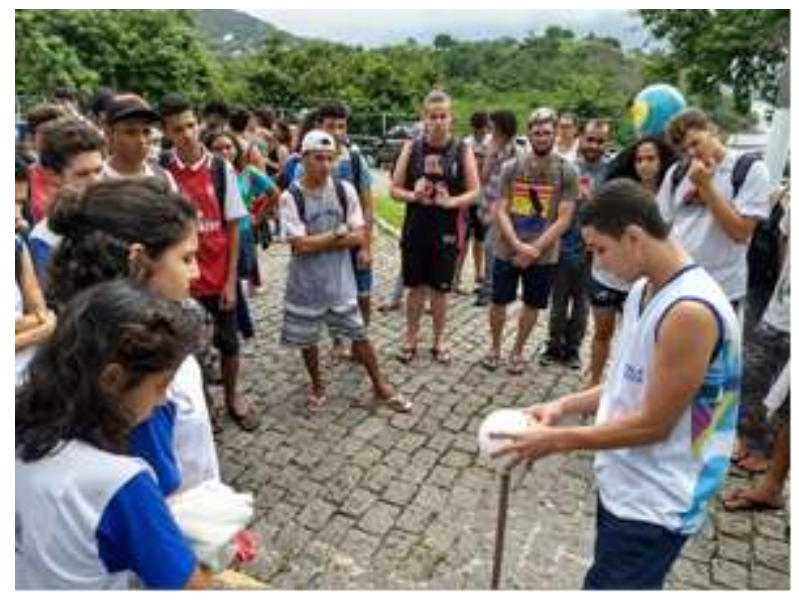

Figura 4:Apresentação do Sistema Solar à comunidade escolar

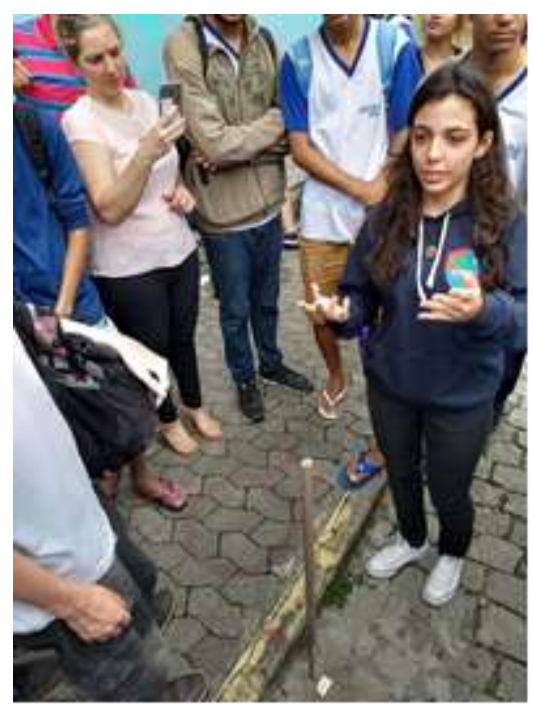

Figura 5:Apresentação do Sistema Solar à comunidade escolar

\section{Discussão e conclusão}

Observou-se que no primeiro Sistema Solar os alunos tinham certa noção de tamanho, mas não sabiam compará-los. Quanto as distâncias entre os planetas e o Sol, verificamos uma forte evidenciação das figuras provenientes dos livros ou de certas imagens midiáticas. Uma das causas dessa situação, talvez seja o que a citação abaixo. Segundo LANGHI (2004):

O panorama geral histórico do ensino da Astronomia no Brasil demonstra o quanto esta Ciência tem se afastado gradualmente dos currículos escolares, a tal ponto de praticamente inexistir em cursos de formação de professores, notadamente de Ensino Fundamental e dos anos iniciais. A existência desta deficiência na formação do docente geralmente implica em geração de dificuldades neste tema durante o seu ensino de Ciências para estudantes (pg.7).

Ainda podemos citar LEITE (2002):

[...] o ensino e a aprendizagem em Astronomia feita através do livro didático não apresentam bons resultados [...] (pg.70)

Os alunos indicaram uma concepção prévia do Sistema Solar sem noção das dimensões de distância e de tamanho. Segundo BISCH (1998) um certo realismo ingênuo.

$\mathrm{Na}$ Teoria da Aprendizagem Significativa (TAS), segundo MOREIRA (1999):

"Os subsunçores existentes na estrutura cognitiva podem ser abrangentes e bem desenvolvidos ou limitados e pouco desenvolvidos [...]"

Podemos dizer, na concepção da TAS, que os alunos apresentaram concepções prévias limitadas e pouco abrangentes.

Com a aplicação da atividade estruturada, verificou-se uma mudança das concepções prévias dos alunos, a nova informação "distância e tamanho dos planetas em escala real" relacionou-se com um aspecto já existente "distância e tamanho pouco abrangente". Podemos dizer, que o conhecimento prévio dos alunos se aproximou do conhecimento científico. Para evidenciar o fato, apresento a fala do aluno 1: "Caramba! Essas distâncias são muito maiores do que tinha visto". Outro vislumbre dos estudantes, foi o fato dos grandes "vazios" entre os planetas. Segundo o aluno 2: "De Marte para Júpiter, é muito longe!".

Na comparação das dimensões dos sistemas solares, a fala do aluno 3 , de espanto, demonstra bem o quanto de informações foram trocadas durantes os debates. Segundo o aluno 3: "Tudo que eu tinha visto, não tem nada haver. A coisa é muito maior!".

De forma geral, quando comparadas as informações que os estudantes davam no Sistema Solar inicial e o que eles apresentaram à comunidade escolar, juntamente com as suas novas análises nos debates, verificamos que estes alunos adquiriram conceitos novos e mais próximos dos conceitos científicos. Os 
subsunçores ficaram mais fortalecidos, e qualquer informação a respeito do Sistema Solar, possivelmente, serão "ancoradas" nos subsunçores mais fortalecidos e com maior facilidade.

Outra conclusão é que, aparentemente, houve acerto na escolha da atividade estruturada desenvolvida com os alunos, baseada na área da Educação em Astronomia. Ainda podemos citar além do avanço no conhecimento gerado, o aumento do interesse dos estudantes com relação à Astronomia.

\section{Referências}

\section{Teses ou dissertações}

[1] BISCH, Sérgio Mascarello. Astronomia no Ensino Fundamental: Natureza e Conteúdo do Conhecimento de Estudantes e Professores. São Paulo: USP, 1998, 301p. Tese de Doutorado, Programa de PósGraduação em Educação, Faculdade de Educação, Universidade de São Paulo. São Paulo, 1998. Disponível em: <http://www.btdea.ufscar.br/arquivos/td/1998_BISCH_T _USP.pdf>.

[2] LEITE, Cristina. Os professores de Ciência e suas formas de pensar a Astronomia. São Paulo: USP, 2002, 160p. Dissertação de Mestrado, Instituto de Física, Faculdade de Educação da Universidade de São Paulo, 2002.
[3] SILVA, T. P. Nossa Posição no Universo: uma proposta de sequência didática para o Ensino de Astronomia no Ensino Médio. Vitória: UFES, 2015, 160p. Dissertação de Mestrado apresentada ao Programa de Pós-Graduação em Ensino de Física do Centro de Ciência Exatas da Universidade Federal do Espírito Santo. Disponível em http://www.btdea.ufscar.br/arquivos/td/2015 SILVA D UFES.pdf.

\section{Artigos}

[4] LANGHI, Rodolfo. Educação em Astronomia: da revisão bibliográfica sobre concepções alternativas à necessidade de uma ação nacional. Caderno Brasileiro de Ensino de Física. Volume 28, № 2. 2011.

[5] LANGHI, Rodolfo. Ideias de Senso Comum em Astronomia. Observatórios Virtuais-Ideias de Senso Comum. 2004.

\section{Livros}

[6] MOREIRA, M. A. Teorias de aprendizagem. São Paulo: Editora Pedagógica e Universitária, 1999. 201 p.

[7] MOREIRA, M. A. Aprendizagem Significativa. Brasília: Editora da UnB. 1999. 129 p. 